\title{
Pesticides-induced carcinogenic \& neurotoxic effect on human
}

\begin{abstract}
Human exposure to pesticides is a worldwide major health problem. In modern age, the use of pesticides on crops has developed most dangerous diseases and some of them are very harmful. Major use of pesticides on crops and direct exposure to them gives rise to neurotoxicity and also causes carcinogenic effect on human beings. A large number of research proved that pesticides have very harmful effects in both conditions whether chronic or direct exposure. In this review, we discussed about the harmful neurotoxic and carcinogenic effects of pesticides on human health. Farmers are more prone to neurotoxicity and carcinogenicity rather than consumers. But some strong pesticides like organophosphates and organochloro have powerful effects that resides in crops for too long and consumers intake them indirectly. Contact to these compounds during initial fetal growth can origin brain injury at doses much lesser than those affecting adult brain function. Hazards of these chemicals are so powerful that also causes damage to environment and through air human's intake them.
\end{abstract}

Volume 7 Issue 5 - 2019

\author{
Himanshu Yadav,' Mahipal Singh Sankhla, ${ }^{2}$ \\ Rajeev Kumar ${ }^{3}$ \\ 'Student of M.Sc. Forensic Science, Galgotias University, India \\ ${ }^{2}$ Research Scholar, Division of Forensic Science Galgotias \\ University, India \\ ${ }^{3}$ Associate Professor, Division of Forensic Science, Galgotias \\ University, India
}

Correspondence: Mahipal Singh Sankhla, Research Scholar, Division of Forensic Science Galgotias University, India, Emailmahipal4n6@gmail.com

Received: July 12, 2019 | Published: September 04, 2019

Keywords: pesticides, human, risk, health, hazardous, etc

\section{Introduction}

An inclusive term Pesticides that comprehends insecticides, miticides, nematicides, fungicides, herbicides, algaecides, fumigants, vertebrate poisons and the rest. Pesticides are synthetics accustomed for slaying or controlling objectionable insects, diseases on plants, weeds, slugs and snails, birds, and vertebrate mammals concerned as pests such as rodents. ${ }^{1}$ There are only three main and leading categories of insecticides are organophosphates, organochlorines and carbamates. In developing nations, the pesticide vulnerability is more comprehensive and upward than in industrialized nations. ${ }^{2}$ The comprehensive utility of pesticides and its virtual impacts on humanbeing health is a rising trouble. ${ }^{3}$ Neurotoxicity can be demonstrate as critical signs and symptoms over eminent acute denunciation, or by additional elusive effects upon persistent vulnerability to small doses. ${ }^{4}$ There is further a risk consideration in the evolution of neurodegenerative diseases, like Parkinson's disease due to the exposure of pesticides. ${ }^{5}$

In European origin, almost 300 distinct pesticides have been proclaimed as toxins or contaminants of food products. ${ }^{6}$ Equal to 50 percent of fruits, vegetables and cereals grown in the European Union are known to comprise pesticide deposits. ${ }^{6}$ It is notable now that a suggestive proportion of pesticides are carcinogenic; for illustration, $18 \%$ of all insecticides and $90 \%$ of all fungicides were ground to be carcinogenic. ${ }^{7}$ Even it is also well known that pesticides are peculiarly toxic to the young and that pesticide dregs persist for long periods of time. ${ }^{8}$ The quality of water and its reservoir are undeviatingly contaminated by the chemical comprising pesticides. The pesticides would be hazardous for agriculture ecosystem if they are in enormous quantity or inadequately handled. ${ }^{9}$ Exclusively $60 \%$ fertilizers are used in the soil other chemicals drained in to soils contaminating the water, cyanobacteria are abundant in contaminated water and surplus phosphate run off prompt to eutrophication. Excess of pesticides enters in the food chain directly and also after combining with river waterdue to flooding, heavy rainfall and excess irrigation. ${ }^{10}$
Countless vegetables and fruits are polluted with these pesticides and they are mortal for human beings. ${ }^{11}$ Several pesticides have been found in drinking water sources at absorption above EPA margin and of virtual threat to human health. ${ }^{12}$ The mechanism of toxicokinetics of pesticides generally entangles a neurotoxic influence: organophosphorous compounds operate via inhibition of central nervous system cholinesterase,$^{13}$ pyrethroids influence the sodium channels of the nerve membrane, holding them accessible for extra than the few milliseconds desired for the procreation of the action ability ${ }^{14}$ organochlorinated compounds in common perform as central nervous system stimulants, but the toxicokinetics dissimilate for the peculiar dynamic components. ${ }^{15}$ Cell signaling negotiated via adenylyl cyclase (AC) is the one potential noncholinergic medium for the developmental neurotoxicity of chloropyriphos, the enzyme that regulates the synthesis of the second messenger cyclic AMP. ${ }^{16}$ The hazards confederated to DDT (dichlorodiphenyltrichloroethane) was made prevalent by the publication of the popular Silent Spring by the biologist Rachel Carson in $1962 .{ }^{17}$ Human health is directly affected by the consumption of polluted water, fish, fruits, vegetables, and plants etc. which are the main sources of food for humans. Studies show that agricultural activities that have released hazardous and toxic constituents in thereby, led to contamination of drinking water in these areas. ${ }^{18}$

\section{Neurotoxicity of pesticides}

Neurotoxicity can be defined as any contrary influence on the central or peripheral nervous system induced by chemical, biological or physical agents. ${ }^{4}$ Nonetheless, cancer has been the foremost concern about chemicals, the neuro-behavioural effects of denunciation to chemicals remains a subject-matter of considerable present concern and importance..$^{19}$ Formerly, it was contemplation that the antagonistic effects on brain development reflected the alike elemental mechanism that underlies integral noxiousness, particularly, cholinesterase inhibition and resultant cholinergic hyperstimulation. ${ }^{20}$ Though, exhibit compilation over the elapsed decade entangles 
a host of other mechanisms that rely alternately upon the direct targeting of incidents peculiar to the developing brain. ${ }^{21}$ Accretion of acetylcholine at cholinergic synapses caused by inhibition of $\mathrm{AChE}$ (acetylcholinesterase), bring about over-stimulation of muscarinic and nicotinic receptors. In addition, acetylcholine has crucial functions during brain development. ${ }^{22}$ Developmental neurotoxicity was proposed by an anthropological review of two alike groups of symptomless, Yaqui children aged 4-5 years in Mexico. ${ }^{23}$

Those with eminent denunciation to a mix of pesticides, including organophosphates, had diminished short-term memory, hand-eye coordination, and drawing ability, when in fact obscured children of the same tribe revealed ordinary development. ${ }^{23}$ Similarly, preschool children from agricultural fraternities in the USA revealed imperfect accomplishment on motor speed and latency than did those of urban fraternities. ${ }^{24}$ In addition to the acute cholinergic syndrome, OPs may also a reason of intermediate syndrome, which is witnessed in $20-50 \%$ of acute OP poisoning cases. ${ }^{25}$ The intermediate affliction is not a direct effect of AChE inhibition, and its explicit elemental mechanisms are anonymous, however it may terminate from nicotinic receptor desensitization by reason of elongated cholinergic stimulation. ${ }^{26}$ The CNS manifestations of OP intoxication consists convulsions, speech disorders, insomnia, drowsiness, coma, anxiety, irritability, depression, impaired memory, and personality disorders. ${ }^{27}$ The cholinergic symptoms on the CNS consist dizziness, mental confusion, headache, weakness, convulsions and coma. ${ }^{28}$ Some OPs have been connected with peripheral nerve diseases that emerges a few weeks after a intoxication incident.

The materialization of Organophosphate-induced delayed polyneuropathy (OPIDN) seems to accompany the phosphorylation and sequential maturing of an enzyme in axons called as neuropathy target esterase. ${ }^{29}$ Signs and symptoms consist shivering of the hands and feet, accompanied by sensory loss, advanced muscle impairment and flaccidity of the distal skeletal muscles of the lower and upper extremities, and then ataxia, which may happen 2-3 weeks after a single divulgence, when signs of both the acute cholinergic and the intermediate syndromes have depressed. ${ }^{30}$ The mechanism of poisonousness of carbamates is alike to that of OPs, as they also inhibit AChE. ${ }^{4}$ Oximes have been revealed to enhance the lethalness of carbaryl, but may have advantageous manifestations in case of other carbamates, such as aldicarb. ${ }^{31}$ The OP delayed neurotoxicity syndrome arises one to three weeks after divulgence. Earliest manifestations consists paresthesia, hypesthesia, abnormal reflexes, and muscle weakness. ${ }^{32}$

\section{Carcinogenicity of pesticides}

Pesticides causes cancer, there is a substantial body of epidemiological exhibits linking to this fact, and in peculiar to child cancer following from both patriarchal and direct nonage exposures. For leukaemia and brain cancer exhibits are powerful, but there is exhibits also for confederation with non-Hodgkin's lymphoma, neuroblastoma, Ewing's sarcoma (a cancer of bone tissue), and Wilm's tumour (kidney). ${ }^{33}$ Also a number of adult cancers are there linked with denunciation to pesticides along with breast, lung, multiple myeloma, non-Hodgkin's lymphoma, leukaemia, ovary, pancreas, prostate, kidney bladder, stomach, colon, rectal, lip, connective tissue, brain, and testicular. ${ }^{1}$ Of these, at least breast, prostate, and testicular cancer are conceit to have genesis in betimes developmental denunciations to ecological hormone disruptors. ${ }^{34}$ Epidemiological researches have linked an apparel of cancers with all the chief operational categories of pesticides - herbicides, insecticides, fungicides, fumigants - and chemical categories consisting organochlorine (OC), organophosphate (OP), and carbamate insecticides, and phenoxy acid and triazine herbicides. ${ }^{34}$

\section{Discussion}

The use of heavy pesticides should be banned in excess amount and preventions should be taken by regulatory authorities. In fact, lack of knowledge in farmers about the proper dosage, prevention steps and hazardous effects of pesticides also causes problems. A popular organochloro compound named "Endosulfan" was banned by the government because of its very lethal effects in kerala. It induces foetal hazards leading to anatomical changes in children's. These kind of conditions occurs when amount or dosage of pesticides exceeds more than the safe limit. We should control the situation before it gets more horrible.

\section{Conclusion}

i. Many researchers proved that pesticides have tremendous effects on humans. It has been proved that dangerous diseases like cancer are also a byproduct of pesticides exposure directly or indirectly. The effects of heavy organophosphates like Chloropyriphos, Monocrotophos and Methyl Parathion resides in crops for a long term. When we consume that eatable stuff it indirectly enters in the body and causes serious conditions. It has been seen that earlier the diseases like cancer and neurotoxicity was rare. But in recent few years, in the chemical evolution heavy pesticides uses on crops created a terrific situation for human wellbeing. Organochloro are the chlorinated hydrocarbons and causes very lethal damage to the ecosystem and affects the human health through contamination in soil, food, water and also through air via spray of pesticides and particles of chemicals contaminate the air also. Most of the farmers use heavy doses of Nitrobenzene on crops for maximum yield but it is a highly carcinogenic compound. It has been proved that the discarded packing material also contaminate the soil leading to various health problems for us. Even discarded bottles and packets of pesticides packing material in water resources like pond, river etc. also a major factor of contamination of water bodies from pesticides. A product of Carbamates compounds 'Carbofuran' is highly toxic and remains available in crops for three to four months. If we consume the crop before this period, the pesticide content will also be consumed by us indirectly. All categories of pesticides are harmful for human health if it is excessively used.

To resolve the issues generated by extensive use of pesticides, there are following notable measures suggested by researchers:

ii. Use of Organochloro pesticides should be minimized as they are not short-lived in the ecological system, they persist for a long time and causes serious ecological damage.

iii. During the washing of sprayer equipments of pesticides, always make sure the drain water won't contaminate the water reservoirs.

iv. Always read the instruction label of using pesticides carefully because it contains the environmental hazards section.

v. Always wear mask and gloves while spraying pesticides. Never spray in the opposite direction of wind and also keep the nozzle downward towards the earth surface. 
vi. Never use the pesticides while raining.

vii. Never throw the pesticides containers in the water reservoirs, discard them appropriately.

viii. Wash the vegetables and fruits properly before consumption so the effect of pesticides concentration can be minimized.

ix. Don't use extremely toxic pesticides on eatable crops when they are about to consume (during final term of crops).

\section{Acknowledgments}

None.

\section{Conflicts of interest}

The author declares that there are no conflicts of interest.

\section{References}

1. Meriel Watts. Human Health Impacts of Exposure to Pesticides. Contract ref: 011005 .

2. World Health Organization and United Nations Environment Program Public health impact of pesticides used in agriculture. Geneva. 1990.

3. Binukumar BK, Kiran Dip G. Chronic Exposure to PesticideNeurological, Neurobehavioral and Molecular Targets of Neurotoxicity. Pesticides in modern world-effect of pesticides exposure. 2010.

4. Lucio G Costa, Gennaro Giordano, Marina Guizzetti, et al. Neurotoxicity of pesticides: a brief review. Frontiers in Bioscience. 2008;13(4):1240-1249.

5. Ascherio A, Chen H, Weisskopf MG, et al. Pesticide exposure and risk of Parkinson's disease. Ann Neurol. 2006;60(2):197-203.

6. Monitoring of pesticide residues in products of plant origin in the European Union, Norway, Iceland and Liechtenstein. Commission staff working document. 2005

7. NAS. Regulating pesticides in food. Washington DC, National Academy of Sciences. 1987.

8. Andersson H, Tago D, Treich N. Pesticides and health: A review of evidence on health effects, valuation of risks, and benefit-cost analysis. Adv Health Econ Health Serv Res. 2014;24:203-295.

9. Yonglong $\mathrm{Lu}$, Song S, Wang R, et al. Impacts of soil and water pollution on food safety and health risks in China. Environment International. 2015;77:5-15.

10. Mehtab Haseena, Muhammad FM, Asma J, et al. Water pollution and human health. Environ Risk Assess Remediat. 2017;1(3).

11. Kamble SM. Water pollution and public health issues in Kolhapur city in Maharashtra. Inter J scien \& res public. 2014;4(1):1-6.

12. Hetrick J, Parker R, Pisigan R, et al. Progress report on estimating pesticide concentration in drinking water and assessing water treatment effects on pesticide removal and transformation. FIFRA Scientific Advisory Panel (SAP). 2000.

13. Jeyaratnam J, Maroni M. Organophosphorous compounds. In: Tordoir WF, Maroni M, editors. Health surveillance of pesticide workers. A manual for occupational health professionals. Toxicology. 1994;15-28.

14. He F. Synthetic pyrethroids. In: Tordoir WF, Maroni M, editors. Health surveillance of pesticide workers. A manual for occupational health professionals. Toxicology. 1994;43-50.
15. Tordoir WF, Van Sittert NJ. Organochlorines. In: Tordoir WF, Maroni $\mathrm{M}$, editors. Health surveillance of pesticide workers. A manual for occupational health professionals. Toxicology. 1994;51-8.

16. Aldridge JE, Seidler FJ, Meyer A, et al. Serotonergic Systems Targeted by Developmental Exposure to Chlorpyrifos: Effects during Different Critical Periods. Environ Health Perspect. 2003;111(14):1736-1743.

17. Carson R. Silent Spring. Boston: Houghton Mifflin. 1962.

18. Sankhla MS, Kumari M, Sharma K, et al. Water Contamination through Pesticide \& Their Toxic Effect on Human Health. Int J Res Appl Sci Eng Technol. 2018;6(1):967-970.

19. Walker B. Neurotoxicity in human beings. J Lab Clin Med. 2000;136(3):168-180.

20. Pope $\mathrm{CN}$. Organophosphorus pesticides: do they all have the same mechanism of toxicity? J Toxicol Environ Health B Crit Rev. 1999;2(2):161-181.

21. Barone S, Das KP, Lassiter TL, et al. Vulnerable processes of nervous system development: a review of markers and methods. Neurotoxicology. 2001;21(1-2):15-36.

22. Lauder JM, Schambra UB. Morphogenetic roles of acetylcholine. Environ Health Perspect. 1999;107(Supp1 1):65-69.

23. Guillette EA, Meza MM, Aquilar MG, et al. An anthropological approach to the evaluation of preschool children exposed to pesticides in Mexico. Environ Health Perspect. 1998;106(6):347-353.

24. Rohlman DS, Arcury TA, Quandt SA, et al. Neurobehavioral performance in preschool children from agricultural and non-agricultural communities in Oregon and North Carolina. Neurotoxicology. 2005;26(4):589-98.

25. Senanayake N, Karalliedde L. Neurotoxic effects of organophosphate insecticides. An intermediate syndrome. New Engl J Med. 1987;316(13):761-763.

26. Lotti M. Clinical toxicology of anticholinesterases in humans. In Krieger R, editor. Handbook of Pesticide Toxicology. Academic Press, San Diego. 2001;1043-1085

27. Wadia SR, Sadagopan C, Amin RB, et al. Neurological manifestations of organophosphorus insecticide poisoning. J Neurol Neurosurg Psychiatry. 1974;37:841-847.

28. Costa L. Basic toxicology of pesticides. In: Keifer M, editor. Occupational Medicine: the state of the Art Reviews. Philadelphia: Hanley \& Belfus, Inc. 1997.

29. Johnson MK. A phosphorylation site in brain and the delayed neurotoxic effect of some organophosphorous compounds. Biochem J. 1969;111(4):487-495.

30. Lotti M, Moretto A. Organophosphate-induced delayed polyneuropathy. Toxicol Rev. 2005;24(1):37-49.

31. Ecobichon DJ. Carbamate insecticides. In: Krieger R, editor. Handbook of Pesticide Toxicology. Academic Press, San Diego. 2001;1087-1106.

32. Vora DD, Dastur DK, Braganca BM, et al. Toxic polyneuritis in Bombay due to ortho-cresyl-phosphate poisoning. J Neurol Neurosurg Psychiatry. 1962;25(3):234-242.

33. Infante-Rivard $\mathrm{C}$, Weichenthal $\mathrm{S}$. Pesticides and childhood cancer: an update on Zahm and Ward's 1998 review. J Toxicol Environ Health B Crit Rev. 2007;10(1-2):81-99.

34. Bassil KL, Vakil C, Sanborn M, et al. Cancer health effects of pesticides. Can Fam Physician. 2007;53(10):1704-1711. 